\title{
Design of a Decoupled Six-axis Force Sensor for Powered Lower Limb Exoskeleton
}

\author{
Kai Yang ${ }^{1}$, Shun $\mathrm{Ma}^{2}$, Tianshuo Wang ${ }^{2}$ and Yanhe Zhu² \\ ${ }^{1}$ Post-doctoral Scientific Research Station in Nanjing General Hospital of Nanjing Military Region; \\ ${ }^{2}$ Harbin Institute of Technology, State Key Laboratory of Robotics and System, Harbin, Heilongjiang, \\ China. \\ yhzhu@hit.edu.cn
}

\begin{abstract}
In this paper, we introduce the six-axis force sensor used for detecting the human-machine interaction and predicting the wearer' s movement intention of the lower limbs powered exoskeleton robot, including the mechanical design, parameters determination and finite element simulation. With the help of the sensor, the exoskeleton can conclude the current motion state and forecast the human movement intention, and achieve human-machine following movement and supply power.
\end{abstract}

Keywords: Six-axis force sensor; Mechanical design; Finite element simulation; Exoskeleton.

\section{Introduction}

In recent years, with the development of technology, the exoskeleton robot has been widely applied and developed in military, aerospace, industrial and medical field [1]. The exoskeleton is a wearable auxiliary mechatronic system, combined the intelligence of human with the physical strength of robot [2]. In order to achieve specific functions, the lower limbs powered exoskeleton robot must be able to detect, determine and follow the wearer's movement and movement intention real-timely.

There have been plenty of mature exoskeleton robot currently. The Tsukuba University developed the hybrid assistive limb, which use DC servo motors to drive the knee joint and knee joint. With the help of angle sensors and detecting feet pressure, HAL has achieved to control the motors to move by collecting EMG signal of wearers, perceiving movement intention [3-6]. The lower limbs powered exoskeleton used for the earthquake rescue system developed by HIT (HIT-LEX), is combined with 15 degrees of freedom, and its 6 degrees of freedom are drive by motors. The HIT-LEX can walk with the speed of $1.5 \mathrm{~m} / \mathrm{s}$ and run 4 hours when loading $70 \mathrm{~kg}$ of weight. The HIT-LEX can determine the wearer's movement interaction with help of joint angle sensors, contact sensors of feet and the six-axis force sensors on feet and back. It can balance the friction damping and self-gravity. Under the cooperation of detecting and predicting the wearer's movement intention, balancing inertial force, the wearers will have no sense of weight when wearers wear HIT-LEX and load weight [7, 8].

This paper will introduce the six-axis force sensors used for detecting and predicting the wearer's movement condition and intention.

\section{Structural Design for Six-axis Force Sensor}

For the lower limbs powered exoskeleton robot, we need to detect the human-machine interaction including the three-dimensional force of soles of the feet, the three-dimensional force and the two-dimensional torque of the back without vertical direction torque. Considering the mechanical space limit, we designed the human-machine interaction sensing system as the flat shape on the kinematic end. So we developed a six-axis force sensor to detect the human-machine interaction. As the Fig. 1 shown, there are six-dimensional force detection at the back and feet. About the six-axis force sensor designing, we need to consider the following aspects: the flat shape, the reasonable mechanical limit and the installation with the kinematic end of the exoskeleton robot. 

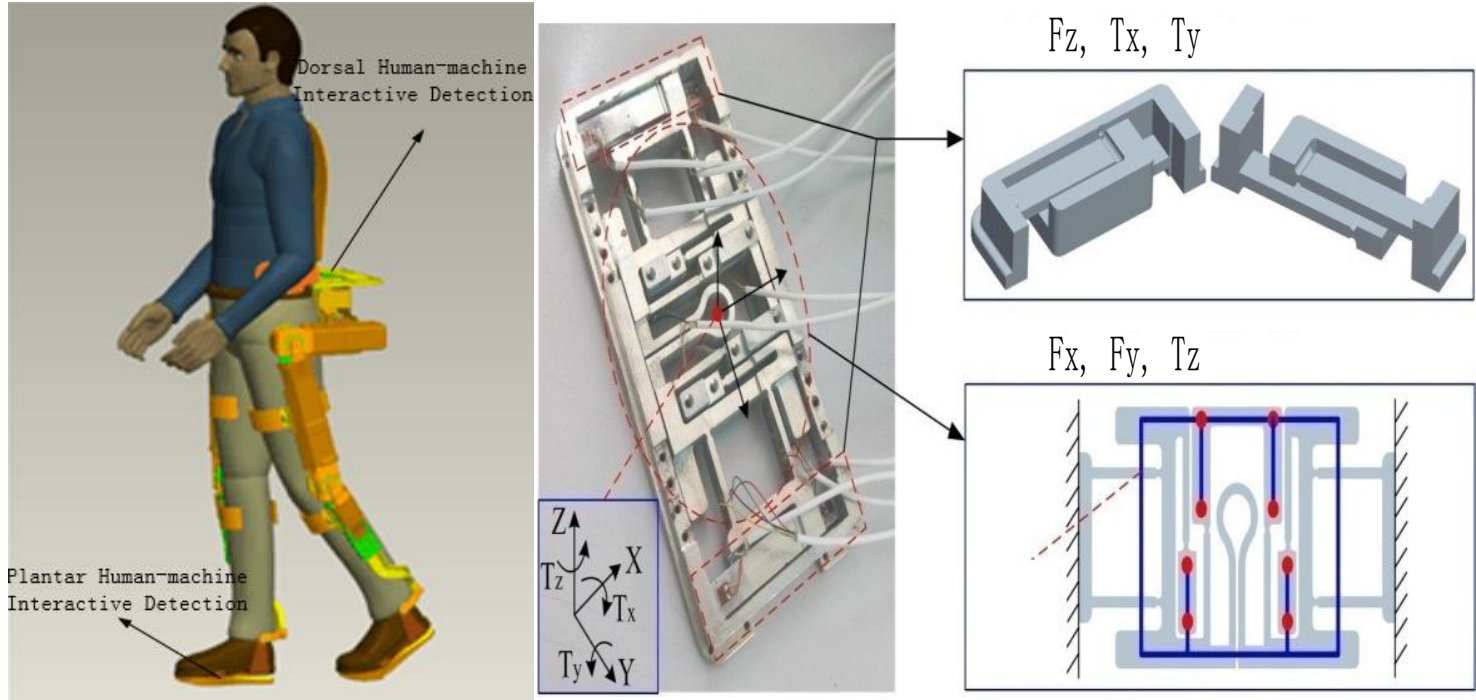

Fig. 1 Arrangement of the six-axis force sensing system

We use the $65 \mathrm{Mn}$ material to make the six-axis force sensor. There are two cantilever girders and four points support principle at both ends of the sensor, and we use the half-bridge circuit to detect the four support force of the support points. So we can get the one-dimensional force and the two-dimensional torque of the human-machine interaction. Its schematic is shown as the Fig. 2.

$$
\begin{aligned}
& F_{Z}=F_{Z 1}+F_{Z 2}+F_{Z 3}+F_{Z 4} \\
& T_{x}=\left(F_{Z 1}+F_{Z 2}-F_{Z 3}-F_{Z 4}\right) \cdot a \\
& T_{y}=\left(F_{Z 1}+F_{Z 3}-F_{Z 2}-F_{Z 4}\right) \cdot b
\end{aligned}
$$

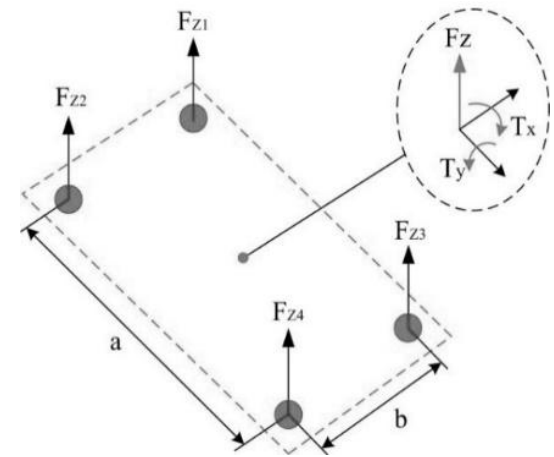

Fig.2 Schematic diagram of four points support at both ends of the sensor

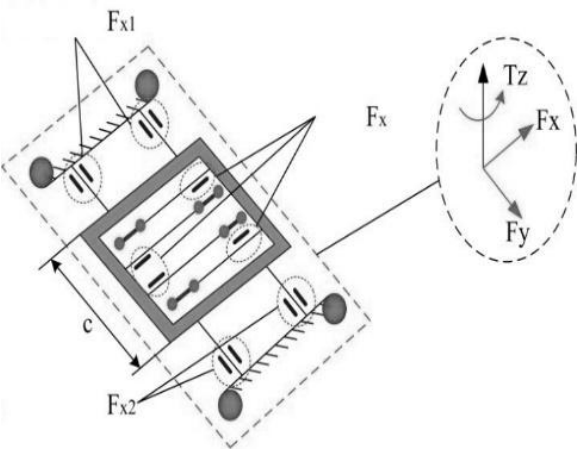

Fig. 3 Schematic diagram of force measuring of the center part

There are eight cantilever girders as the force measuring of the sensor center part. According to the eight cantilever girders, we can get the other two-dimensional force and one-dimensional torque. Its schematic is shown as the Fig. 3. The six-axis force sensor and the schematic of the interaction force detection at kinematic terminals are shown as the Fig. 3.

$$
\begin{aligned}
& F_{x}=F_{x 1}+F_{x 2} \\
& F_{y}=F_{y} \\
& T_{z}=\left(F_{x 2}-F_{x 1}\right) \cdot c
\end{aligned}
$$




\section{Determination of Structural Parameters and Finite Element Simulation for Six-axis Sensor}

In order to minimize the coupling between the dimensions of the six-axis force sensor, we use symmetrical cantilever girders to design the structure of the six-axis force sensor. There should be some deformation on the end of the cantilever, so that we can ensure the safety of the machining and design of the mechanical limit. What's more, for the purpose of increase the flexural modulus of section of the sensitive directions, the length and width of the cantilever girders should be larger. Considering unity design between soles of the feet and back of the exoskeleton, the sizes of the six-axis force sensor are designed $85 \mathrm{~mm} \times 175 \mathrm{~mm}$.

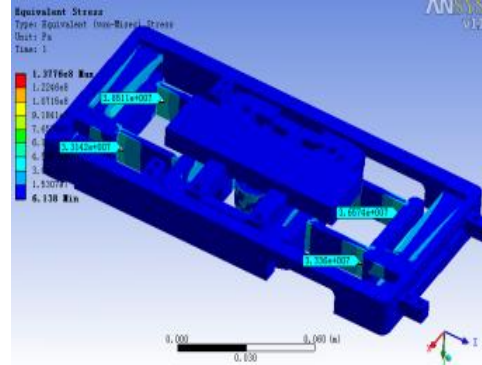

a) $\mathrm{X}$ direction $(50 \mathrm{~kg})$

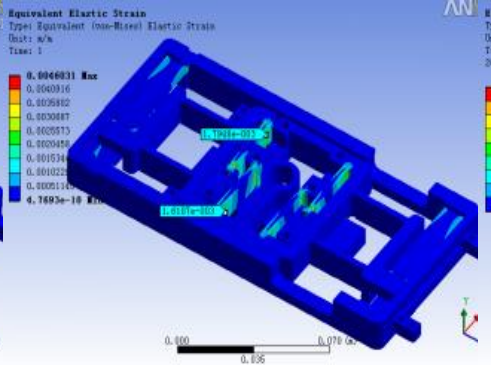

b) $\mathrm{Y}$ direction $(50 \mathrm{~kg})$

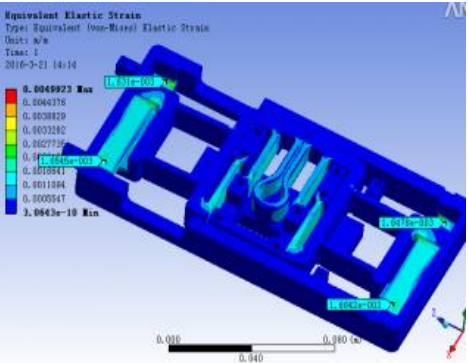

c) $\mathrm{Z}$ direction $(50 \mathrm{~kg})$

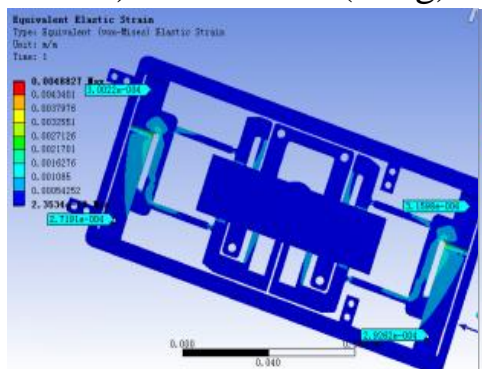

d) $\mathrm{X}$ direction $(16.8 \mathrm{Nm})$

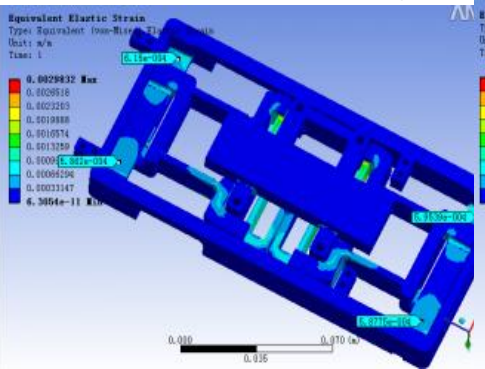

e) $\mathrm{Y}$ direction $(18.5 \mathrm{Nm})$

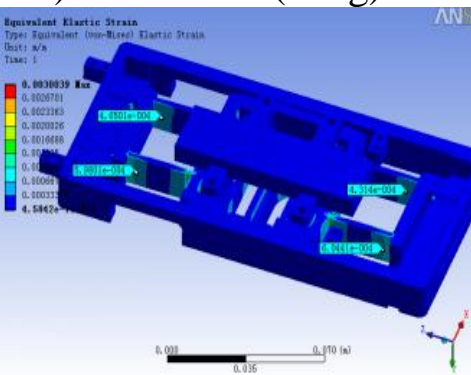

f) $\mathrm{Z}$ direction $(19.5 \mathrm{Nm})$

Fig. 4 Finite element analysis under single force or torque.

Table 1 Finite element analysis result

\begin{tabular}{cccc}
\hline Force/Torque & Maximum Range & Maximum Strain & Maximum Stress \\
\hline$F_{x}$ & $50 \mathrm{~kg}$ & $1.642 \mathrm{~mm} / \mathrm{m}$ & $353.35 \mathrm{MPa}$ \\
$F_{y}$ & $50 \mathrm{~kg}$ & $1.175 \mathrm{~mm} / \mathrm{m}$ & $237.54 \mathrm{MPa}$ \\
$F_{z}$ & $50 \mathrm{~kg}$ & $1.351 \mathrm{~mm} / \mathrm{m}$ & $246.32 \mathrm{MPa}$ \\
$T_{x}$ & $16.8 \mathrm{Nm}$ & $0.623 \mathrm{~mm} / \mathrm{m}$ & $212.61 \mathrm{MPa}$ \\
$T_{y}$ & $18.5 \mathrm{Nm}$ & $0.321 \mathrm{~mm} / \mathrm{m}$ & $345.87 \mathrm{MPa}$ \\
$T_{z}$ & $19.5 \mathrm{Nm}$ & $0.611 \mathrm{~mm} / \mathrm{m}$ & $214.37 \mathrm{MPa}$
\end{tabular}

The sensor should achieve the mechanical limits when the support force of single direction is $50 \mathrm{~kg}$. According to the maximum range of the three-dimensional force, we can determine the gap of the mechanical limits and the maximum range of the three-dimensional torque. We can define the specific size of the maximum ranges by the finite element analysis. The results of the finite element analysis are shown in Table 1 and Fig. 4. It shows that the gaps for protection can ensure the maximum strain of the material is within the allowable range, and the cantilever girders are sensitive to the force directions. At last, we define the maximum ranges of the six-axis force sensor are $50 \mathrm{~kg}$, $50 \mathrm{~kg}, 50 \mathrm{~kg}, 16.8 \mathrm{Nm}, 18.5 \mathrm{Nm}, 19.5 \mathrm{Nm}$. According to the modal analysis, the natural frequency is $450 \mathrm{~Hz}$, which sensitive direction is along the $\mathrm{X}$ axis. Under normal circumstances, we define the $2 / 3$ of the natural frequency as the operating bandwidth of sensors. So the operating bandwidth of the six-axis force sensor we design is $0 \sim 280 \mathrm{~Hz}$. 


\section{Conclusion}

In this paper, we introduce the six-axis force sensor used for detecting the human-machine interaction of the lower limbs powered exoskeleton robot, including mechanical design, parameters determination and analysis simulation. We fix the six-axis force sensor on the end of kinematics, the soles of the feet and the back of the exoskeleton robot. With the help of detecting joint angle, posture and touchdown condition, we can conclude the current motion state and forecast the human movement intention. So as to it can achieve human-machine following movement and supply power.

\section{Acknowledgements}

The work reported in this paper is funded by National High Technology Research and Development Program of China (863 Program) under Grant 2012AA041505 and supported by Self-Planned Task (No. SKLRS 201201 A02) of State Key Laboratory of Robotics and System (HIT). Corresponding author: Yanhe Zhu, State Key Laboratory of Robotics and System, Harbin Institute of Technology, Harbin 150001, China. Email: yhzhu@ hit.edu.cn

\section{References}

[1]. Robert B. Robotic exoskeletons: a review of recent progress [J]. Industrial Robot: An International Journal, 2015, 42 (1): 5-10.

[2]. YANG CANJUN, NIU BIN, CHEN YING. Adaptive neuro-fuzzy control based development of a wearable exoskeleton leg for human walking power augmentation [C] // Proceedings of the 2005 IEEE/ ASME International Conference on Advanced Intelligent Mechatronics, Monterey, California, USA, 2005:24-28.

[3]. H. Satoh, T. Kawabata, Y. Sankai. Bathing care assistance with robot suit HAL [J], 2009 IEEE International Conference on Robotics and Biomimetics (ROBIO), 2009: 498-503.

[4]. A Tsukahara, Y Hasegawa, Y Sankai. Standing-up motion support for paraplegic patient with Robot Suit HAL [J]. 2009 IEEE International Conference on Rehabilitation Robotics, 2009: 211-217.

[5]. H Kawamoto, T Hayashi, T Sakurai, K Eguchi, Y Sankai. Development of single leg version of HAL for hemiplegia [C], 31st Annual International Conference of the IEEE EMBS, Minnesota, USA, 2009: 5038-5043.

[6]. H. Kawamoto, S. Taal, H. Niniss et al. Voluntary motion support control of Robot Suit HAL triggered by bioelectrical signal for hemiplegia [C]. 2010 Annual International Conference of the IEEE Engineering in Medicine and Biology Society (EMBC), 2010:462-466.

[7]. Zhu Y, Zhang G, Zhang C, et al. Biomechanical modeling and load-carrying simulation of lower limb exoskeleton [J]. Bio-Medical Materials and Engineering, 2015, 26 (s1): 729-738.

[8]. Zhang C, Zhu Y, Fan J, et al. Design of a quasi-passive 3 DOFs ankle-foot wearable rehabilitation orthosis [J]. Bio-Medical Materials and Engineering, 2015, 26 (s1): 647-654. 